\title{
Study on A Fault Diagnosis Method of Rolling Element Bearing Based on Improved ACO and SVM Model
}

\author{
Wu Deng ${ }^{1,2,3,4,5}$, Xiumei Li ${ }^{1,2}$ and Huimin Zhao ${ }^{1,2,3,4,5^{*}}$ \\ ${ }^{1}$ Software Institute, Dalian Jiaotong University, Dalian 116028 China \\ ${ }^{2}$ The State Key Laboratory of Mechanical Transmissions, Chongqing \\ University, Chongqing 400044 China \\ ${ }^{3}$ Traction Power State Key Laboratory of Southwest Jiaotong \\ University, Chengdu 610031 China \\ ${ }^{4}$ Guangxi Key Laboratory of Hybrid Computation and IC Design Analysis \\ (Guangxi University for Nationalities), Nanning 530006 China \\ ${ }^{5}$ Provincial Key Laboratory for Computer Information Processing Technology \\ , Soochow University, Suzhou 215006 China \\ dw7689@gmail.com
}

\begin{abstract}
The vibration signal is nonstationary and it is difficult to acquire the sample with typical fault. An improved ACO algorithm based on adaptive control parameters is introduced into SVM model to propose a new fault diagnosis (IMASFD) method in this paper. In the IMASFD method, the EMD method is used to decompose fault vibration signal into IMF components, the energy of IMF components is selected to construct the fault feature vectors. Then the adaptive controlling pheromone strategy, adaptive controlling stochastic selection threshold strategy and dynamic evaporation rate strategy are used to improve the basic ACO algorithm. The improved ACO algorithm is used to optimize the parameters of SVM model in order to obtain the optimal values of parameter combination in the SVM model. And a new fault diagnosis (IMASFD) method is proposed. Finally, the proposed IMASFD method is applied to the test data from bearing data center of CWRU. The experimental results show that the proposed method can accurately and effectively realize high precision fault diagnosis of rolling bearing, and has strong robustness and generalization ability, provides an effective method for realizing fault diagnosis of rolling bearing.
\end{abstract}

Keywords: Fault diagnosis; improved ant colony optimization algorithm; support vector machine; parameter optimization; rolling bearing

\section{Introduction}

Fault is an abnormal operating state that takes place during an emergency. It is the consequences of combinations of events that unexpectedly occur at the same time. It could result from process variables, process components, or even basic control systems. Fault diagnosis is a technology for knowing and mastering the equipment state in the operation process, determining the normal or abnormal of the overall or partial, early detecting the fault and its reason and forecasting the development trend of fault[1]. So the efficient and accurate fault diagnosis is an important technology for ensuring the reliability and performance of the operation system. The traditional fault diagnosis method was performed by human operator, this method was essential to quickly estimate the fault component.

With the continuous development of technology and economy, the system complexity is increasing, and it is increasingly important to have techniques to help 
human operators in accessing reliable information and making decisions. A lot of fault diagnosis methods were proposed [2], such as state estimation method, parameter estimation method, equivalent space method, Bayes, wavelet analysis and so on. Because the fault diagnosis need high dependability and accuracy, these methods exist low diagnosis precision and speed. In recent decades, various intelligent fault diagnosis methods have been proposed for improving the diagnosis precision and speed [3], such as artificial neural networks (ANN), expert system (ES), rough sets (RS), Petri Net, genetic algorithm (GA), ant colony optimization (ACO) algorithm, support vector machine (SVM), and other new algorithms. Chrissanthi [4] proposed a survey of recent research work in online expert systems for fault detection and diagnosis in technical processes. Liu et. al. [5] proposed a novel method based on wavelet packet transform and rough sets theory for fault diagnosis of gearbox. Nguyen et. al. [6] proposed a genetic algorithm-based dimensionality reduction approach for reliable low-speed rolling element bearing fault diagnosis by exploiting both inter-class separability and intra-class compactness. Each fault diagnosis method has high dependability, accuracy and speed. Yuan and Chu [7] proposed a new multi-class classification of SVM named 'one to others' algorithm is presented to solve the multi-class recognition problems. But for the large-scale complicated system, each method still can't effectively implement diagnosis with high precision and speed. So many researchers proposed a lot of hybrid intelligent fault diagnosis methods based on fusing or combining the different intelligent optimization algorithms in recent years. Yuan and Chu [8] proposed a fault diagnosis method based on support vector machines and artificial immunisation algorithm. Widodo and Yang [9] proposed establishing intelligent system for faults detection and classification of induction motor using wavelet support vector machine (W-SVM). Fei and Zhang [10] proposed support vector machine with genetic algorithm (SVMG) to apply to fault diagnosis of a power transformer, in which genetic algorithm (GA) is used to select appropriate free parameters of SVM. Zhang and Randall [11] proposed a algorithm to design the parameters for optimal resonance demodulation using the combination of fast kurtogram for initial estimates, and a genetic algorithm for final optimization. Lin et. al. [12] proposed a genetic algorithm-Tabu search (GATS) method for the purpose of fault diagnosis of power systems. Azadeh et. al. [13] proposed a unique flexible algorithm for classifying the condition of centrifugal pump based on support vector machine hyper-parameters optimization and artificial neural networks (ANNs) which are composed of eight distinct steps. Chen et al. [14] proposed a novel intelligent diagnosis model based on wavelet support vector machine (WSVM) and immune genetic algorithm (IGA) for gearbox fault diagnosis. Zhang and Zhou [15] proposed a novel procedure based on ensemble empirical mode decomposition (EEMD) and optimized support vector machine (SVM) for multi-fault diagnosis of rolling element bearings. Wang et. al. [16] proposed a noise-based intelligent method based on the techniques of HilbertHuang transform (HHT) and support vector machine (SVM) for engine fault diagnosis (EFD). Yao et. al.[17] focused on time-frequency feature parameters and genetic algorithm neural network techniques in fault diagnosis of railway rolling bearings. Chen et. al.[18] proposed a novel intelligent fault diagnosis model based on multi-kernel support vector machine (MSVM) with chaotic particle swarm optimization (CPSO) for roller bearing fault diagnosis. Pellaco et al. [19] designed and operated a supervised classification system based on quantitative model of the plant together with a support vector machine. Su et al. [20] proposed a multi-fault diagnosis method for rotating machinery based on orthogonal supervised linear local tangent space alignment (OSLLTSA) and least square support vector machine (LS-SVM). Jegadeeshwaran and Sugumaran[21] proposed a on-line condition monitoring by using machine learning approach as a 
possible solution to such problems. Lu et al. [22] proposed a novel dominant feature selection method using a genetic algorithm with a dynamic searching strategy. Kang et al. [23] proposed a highly reliable fault diagnosis approach based on individually trained support vector machines with kernel discriminative feature analysis for low-speed bearings. Yang et al. [24] proposed a gear fault diagnosis based on support vector machine and artificial bee colony algorithm. The fault features were extracted based on the EEMD and the kernel function.

Ant colony optimization (ACO) algorithm [25] is a metaheuristic inspired by the behavior of real ants in their search for the shortest path to food sources. It has the characteristics of robustness, intelligent search and distributed computing and so on, but it exists the low search efficiency and convergence speed, and easy falling into the stagnation and local optimum. Support vector machine (SVM) [26] is one of the most popular tools in bioinformatics for a supervised machine learning methods based on structural risk minimization. But the parameters of the SVM model have a great effect on the performance. So an improved ACO algorithm based on adaptive control parameters is proposed to optimize the kernel parameter $\sigma$ and penalty coefficient $C$ of SVM in order to obtain the optimal values of parameter combination. And a high-precision SVM model based on improved ACO algorithm is proposed, called MACOSVM in this paper.

The rest of this paper is organized as follows. Section 2 briefly introduces the SVM model. Section 3 proposed an improved ACO algorithm based on adaptive control parameters. Section 4 presents a fault diagnosis method of rolling element bearing based on improved ACO and SVM model. In this section, the idea and the steps of the IMASFD method are introduced in detail. Section 5 applies the IMASFD method for rolling element bearing case. Finally, the conclusions are discussed in Section 6.

\section{SVM Model}

Support vector machine (SVM) introduced by Vapnik, is one of the popular tools for a supervised machine learning method based on structural risk minimization. The basic characteristic of the SVM model is to map the original nonlinear data into a higherdimensional feature space where a hyperplane is constructed to bisect two classes of data and maximize the margin of separation between itself and those points lying nearest to the support vectors.

The given the training sample is $S=\left\{\left(x_{i}, y_{i}\right) \mid i=1,2,3, \cdots, m\right\}, m$ is the number of samples, the set $\left\{x_{i}\right\} \in R_{n}$ represents the input vector, $y \in\{-1,1\}$ indicates the corresponding desired output vector, the input data is mapped into the high dimensional feature space by using nonlinear mapping function $\phi(\bullet)$. Then the existed optimal classification hyperplane must meet the following conditions:

$$
\left\{\begin{array}{l}
\omega^{T} x_{i}+b \geq 1, \quad y_{i}=1 \\
\omega^{T} x_{i}+b \leq-1, y_{i}=-1
\end{array}\right.
$$

where $\omega$ is Omega vector of superplane, $b$ is offset quantity. Then the classification decision function is described as follow:

$$
f\left(x_{i}\right)=\operatorname{sgn}\left(\omega^{T} x_{i}+b\right)
$$

The classification model of the LS-SVM is described by the optimization function $\min _{\omega, \xi, b} J\left(\omega, \xi_{i}\right)$ : 


$$
\begin{aligned}
& \min _{\omega, \xi, b} J\left(\omega, \xi_{i}\right)=\frac{1}{2} \omega^{T} \omega+\frac{1}{2} \gamma \sum_{i=1}^{m} \xi_{i}^{2} \\
& \text { s.t. } \quad y_{i}\left[\omega^{T} \phi\left(x_{i}\right)+b\right]=1-\xi_{i}, i=1,2,3, \cdots, m
\end{aligned}
$$

where $\xi_{i}$ is slack variable, $b$ is offset, $\omega$ is support vector, $\xi=\left(\xi_{1}, \xi_{2}, \cdots, \xi_{m}\right), \gamma$ is classification parameter to balance the fitness error and model complexity.

The optimization problem transforms into its dual space. Lagrange function is introduced to solve it. The corresponding optimization problem of the LS-SVM model with Lagrange function is shown:

$$
L(\omega, b, \xi, \alpha)=\frac{1}{2} \omega^{T} \omega+\frac{1}{2} \gamma \sum_{i=1}^{m} \xi_{i}^{2}-\sum_{k=1}^{m} \alpha_{i}\left\{y_{i}\left[\omega^{T} \phi\left(x_{k}\right)+b\right]-1+\xi_{i}\right\}
$$

where $\alpha_{i}$ is the Lagrange multiplier, and $\alpha_{i} \geq 0(i=1,2,3 . \cdots, m)$. The optimal conditions are described as follow:

$$
\left\{\begin{array}{l}
\frac{\partial L}{\partial \omega}=0 \Rightarrow \omega=\sum_{i=1}^{m} \alpha_{i} y_{i} \phi\left(x_{i}\right) \\
\frac{\partial L}{\partial b}=0 \Rightarrow \sum_{i=1}^{m} \alpha_{i} y_{i}=0 \\
\frac{\partial L}{\partial \xi_{i}}=0 \Rightarrow \alpha_{i}=\gamma \xi_{i} \\
\frac{\partial L}{\partial \alpha_{i}}=0 \Rightarrow y_{i}\left(\omega^{T} \phi\left(x_{i}\right)+b\right)-1+\xi=0_{i}
\end{array}\right.
$$

The following linear equation is obtained:

$$
\left[\begin{array}{ll}
0 & L^{T} \\
L & \Omega+\gamma^{-1} I
\end{array}\right]\left[\begin{array}{l}
b \\
\alpha
\end{array}\right]=\left[\begin{array}{l}
0 \\
Y
\end{array}\right]
$$

where $Y=\left[y_{1}, y_{2}, \cdots, y_{m}\right]^{T} \in R^{m}, L \in R^{m}$ is vector of the element $m$, then $Y^{T}=\left[y_{1}, y_{2}, \cdots, y_{m}\right], I$ is unit matrix, $I_{m}=[1,1, \cdots, 1]^{T}, \alpha=\left[\alpha_{1}, \alpha_{2}, \cdots, \alpha_{m}\right]^{T}$, $\Omega=\left[\Omega_{i j}\right]_{m \times m}, \Omega_{i j}=y_{i} y_{j} K\left(x_{i}, x_{j}\right)$. Then the classification decision function is described:

$$
f\left(x_{i}\right)=\operatorname{sgn}\left(\sum_{i=1}^{m} \alpha_{i} y_{i} K\left(x, x_{i}\right)+b\right)
$$

Because the selection of different kernel function can construct SVM model with different performance, it is very important to select the kernel function for constructing a good performance of the SVM model. There have several common kernel functions, such as linear kernel function, polynomial kernel function, radial basis kernel function (RBF), Sigmoid kernel function and so on. In this paper, the SVM model is used to classify the complex data. Due to the RBF with good learning ability, simple form, symmetry radial, good smoothness and analyticity, the RBF is widely applied to 
classify the data with the low dimension, high dimension, small sample, and large sample [27-29]. The RBF is shown as follow:

$$
K\left(x, x_{i}\right)=\exp \left[-\left(x-x_{i}\right)^{2} / 2 \sigma^{2}\right]
$$

Kernel width $\sigma$ and penalty coefficient $C$ is two important parameters in the SVM model. Their selected values directly influences the learning ability and generalization performance.

\section{Improved ACO algorithm}

\subsection{ACO algorithm}

Ant colony algorithm (ACO) was introduced by Dorigo. The ACO is a metaheuristic inspired by the behavior of real ants in their search for the shortest path to food. The ACO algorithm consists of a number of cycles (iterations) of solution construction. In each iteration, a number of ants construct complete solutions by using heuristic information and the collected experiences of previous groups of ants. These collected experiences are represented by the pheromone trail which is deposited on the constituent elements of a solution. Small quantities are deposited during the construction phase while larger amounts are deposited at the end of each iteration in proportion to solution quality. Pheromone can be deposited on the components and/or the connections used in a solution depending on the problem.

In the ACO algorithm, the ACO algorithm simulates the optimization of ant foraging behavior. The procedure of the ACO algorithm is illustrated in Fig.1.

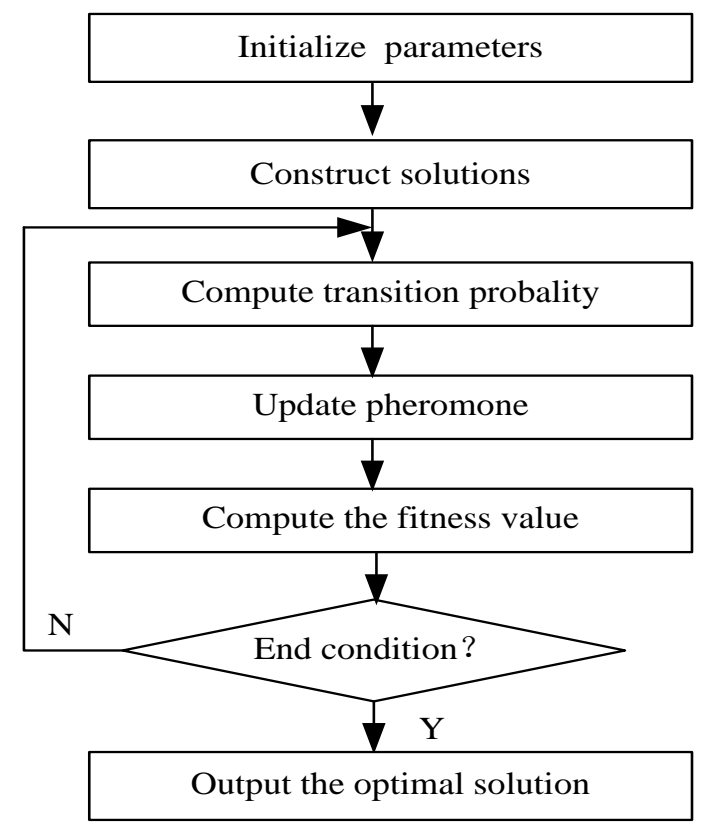

Fig.1. The Flow Of The ACO Algorithm

The procedure of pheromone update rule is shown as follows:

(1) The transition rule

In the route, the $\mathrm{k}^{\text {th }}$ ant starts from city $\mathrm{r}$, the next city $\mathrm{s}$ is selected among the unvisited cities memorized in $J_{r}^{k}$ according to the following expression: 


$$
s=\underset{u \in J_{r}^{k}}{\arg \max }\left[\tau_{i}(r, u)^{\alpha} \cdot \eta(r, u)^{\beta}\right] \text { if } q \leq q_{0}(\text { Exploitation })
$$

To visit the next city s with the probability $p_{k}(r, s)$,

$$
p_{k}(r, s)\left\{\begin{array}{ll}
\frac{\tau(r, s)^{\alpha} \cdot \eta(r, s)^{\beta}}{\sum_{u \in J_{r}^{k}}^{\tau} \tau(r, u)^{\alpha} \cdot \eta(r, u)^{\beta}} & \text { if } s \in J_{r}^{k} \\
0 & \text { otherwise }
\end{array} \text { if } q>q_{0}\right. \text { (Bias Exploitation) }
$$

In two formula, $p_{k}(r, s)$ is the transition probability, $\tau(r, u)$ is the intensity of pheromone between city $\mathrm{r}$ and city $\mathrm{u}$ in the $\mathrm{i}^{\text {th }}$ group, $\eta(r, u)$ is the length of the path from city $\mathrm{r}$ to city $\mathrm{u}, J_{r}^{k}$ is the set of unvisited cities of the $\mathrm{k}^{\text {th }}$ ant in the $\mathrm{i}^{\text {th }}$ group, the parameter $\alpha$ and $\beta$ are the control parameters, $\mathrm{q}$ is a uniform probability $[0,1]$.

(2) The pheromone update rule

In order to improve the solution, the pheromone trails must be updated. Trail updating includes local updating and global updating. The local trail updating formula is given by:

$$
\tau(r, u)=(1-\rho) \tau(r, s)+\sum_{k=1}^{m} \Delta \tau_{k}(r, s)
$$

In the formula (3), $\rho(0<\rho<1)$ is the pheromone trial evaporating rate. $\Delta \tau_{k}(r, s)$ is the amount of pheromone trail added to the edge $(\mathrm{r}, \mathrm{s})$ by ant $k$ between time $t$ and $\mathrm{t}+\Delta \mathrm{t}$ in the tour. It is given:

$$
\Delta \tau_{k}(r, s)= \begin{cases}\frac{Q}{L_{k}} & (\mathrm{r}, s) \in \pi_{k} \\ 0 & \text { otherwise }\end{cases}
$$

where $Q$ is a constant parameter, $L_{k}$ is the distance of the sequence $\pi_{k}$ toured by ant in $\Delta t$.

\subsection{Improved ACO Algorithm based on Adaptive Control Parameters}

\subsubsection{Adaptive Controlling Pheromone}

In the traditional ACO algorithm, a fixed amount of pheromone is used to update the pheromone on the path. This updating strategy ignores the distribution characteristics of solution and is prone to stagnation and slow convergence speed. So adaptive adjusting pheromone strategy is used to make relatively uniform distribution of pheromone and effectively solve the contradiction between expanding search and finding optimal solution in order to search for the local optimal solution in this paper.

The real variable function $Q(t)$ is used to replace the constant of pheromone intensity $Q$ in the adjusting pheromone $\Delta \tau_{i j}^{k}=Q / L_{K}$ in order to balance the exploration and exploitation between the random search of ant and the evocation function of information under the pheromone evaporation or increasing in the random search process. The adaptive adjusting pheromone expression is given:

$$
\Delta \tau_{i j}^{k}(t)=Q(t) / L_{K}
$$




$$
Q(t)= \begin{cases}Q_{1} & t \leq T_{1} \\ Q_{2} & T_{1}<t \leq T_{2} \\ Q_{3} & T_{2}<t \leq T_{3}\end{cases}
$$

In the adaptive adjusting pheromone strategy, if the obtained optimal solution does not change in a period of time, it shows that the search falls into an extreme point. Then the enforcement mechanism is adopted to decrease the amount of increasing information in order to escape from local minimal value. The amount of pheromone on the optimal path and worst path are reduced in order to avoid falling into local optimal solution in the initial stage of search process. Then the positive feedback of ACO need be appropriately restrained by adding a small amount of negative feedback pheromone in the search process, the goal is to reduce the difference of pheromone and expand the scope of the search.

\subsubsection{Adaptive Controlling Stochastic Selection Threshold}

In the traditional ACO algorithm, the value of stochastic selection threshold $q(t)$ is a random number on $[0,1]$. Because the diversity of the solution is increasing, it will reduce to fall into the local optimum in a certain extent. But it is difficult to control the stochastic selection threshold $q(t)$. So the uniformly distributed random number on $[0,1]$ is used to control the stochastic selection threshold $q(t)$. The expression is given:

$$
q(t+1)=\left\{\begin{array}{l}
q(t) / \mu, q(t) \in(0, \mu) \\
(1-q(t)) /(1-\mu), q(t) \in(\mu, 1)
\end{array}\right.
$$

where $\mu \in(0,1)$.

\subsubsection{Dynamic Evaporation Rate}

The evaporation factor of pheromone $\rho$ in the basic ACO algorithm is a constant. The $\rho$ value directly relates to the global search ability and convergence speed. For large-scale problems, because there exists the evaporation factor of pheromone, the pheromone on the unvisited path will be reduced to close to 0 . This will reduce the global searching ability of ACO algorithm. If the pheromone is too large, the selection probability of visited path will be large, this also will affect the global search ability of ACO algorithm. Therefore, how to set the value of pheromone has become the key to control the pheromone releasing and evaporating. The concept of dynamic evaporation rate is used in this paper. The idea is to set a larger value for dynamic evaporation rate $\rho$ at the beginning of ACO algorithm in order to enhance the global search ability. But with the operation of ACO algorithm, the evaporation rate $\rho$ will continue to decay, so that the ACO algorithm can quickly converge to the optimal solution. The dynamic evaporation factor strategy in ACO algorithm not only increases the global search capability, but also accelerates the convergence in a certain extent.

In order to better explore decay model of evaporation rate, there are three different decay models of curve decay model, line decay model and scale decay model. The curve decay model is selected according to implementing a set of experiments. The expression of curve decay model is described as follow:

$$
\begin{aligned}
& \rho(t)=\frac{T \times\left(\tau_{\max }-\tau_{\min }\right) \times t}{T-1}+\frac{T \times \tau_{\text {min }}-\tau_{\text {max }}}{T-1} \\
& \tau(t)=(1-\rho(t)) \times \tau(t)+\sum_{k=1}^{m} \Delta \tau_{i j}^{k}(t)
\end{aligned}
$$


where $\tau_{\max }$ and $\tau_{\min }$ respectively are the upper and lower of pheromone. $t$ and $T$ respectively refer to the current iteration and the maximum iteration.

\section{A Fault Diagnosis Method of Rolling Element Bearing}

\subsection{The Idea of the Hybrid Method Based Improved ACO and SVM Model}

In the SVM model, the penalty coefficient $C$ is used to adjust the proportion of the confidence range and experience risk in the determined subspace in order to obtain the best learning ability. For the given solving problem, when the penalty coefficient $C$ is smaller, it means that the experience error is smaller. Otherwise, in the SVM model, the complexity is smaller, but the experience risk is larger. The inappropriate value of the penalty coefficient $C$ will cause the poor generalization ability. The kernel parameter $\sigma$ is to reflect the distributional complexity of data sample in the high dimensional space, and determine the complexity of linear classification. If the input sample space is larger, the value of kernel parameter $\sigma$ is larger. If the value of kernel parameter $\sigma$ is smaller, the relationship among support vectors is more relaxed, learning machine is relatively complex, and the generalization ability of SVM cannot be guaranteed. If the value of kernel parameter $\sigma$ is larger, the influence among support vectors is too strong, SVM model is difficult to achieve sufficient accuracy. The influence of solving accuracy of kernel parameter $\sigma$ is larger than the penalty coefficient $C$. However, it is difficult to pre-determine the values of the kernel parameter $\sigma$ and penalty coefficient $C$. So It is critical how to effectively determine the appropriate values of the kernel parameter $\sigma$ and penalty coefficient $C$ in order to improve the generalization ability of SVM model. The improved ACO algorithm based on adaptive control parameters is selected to optimize the kernel parameter $\sigma$ and penalty coefficient $C$, in order to obtain the optimal values of parameter combination. And a high-precision SVM model based on improved ACO algorithm is proposed in this paper. The proposed model uses the improved ACO algorithm to automatically select the appropriate values of the kernel parameter $\sigma$ and penalty coefficient $C$ in the SVM model, in order to obtain the optimal performance of SVM classifier. The optimization process of SVM classifier based on the improved ACO algorithm is shown Fig.2. 


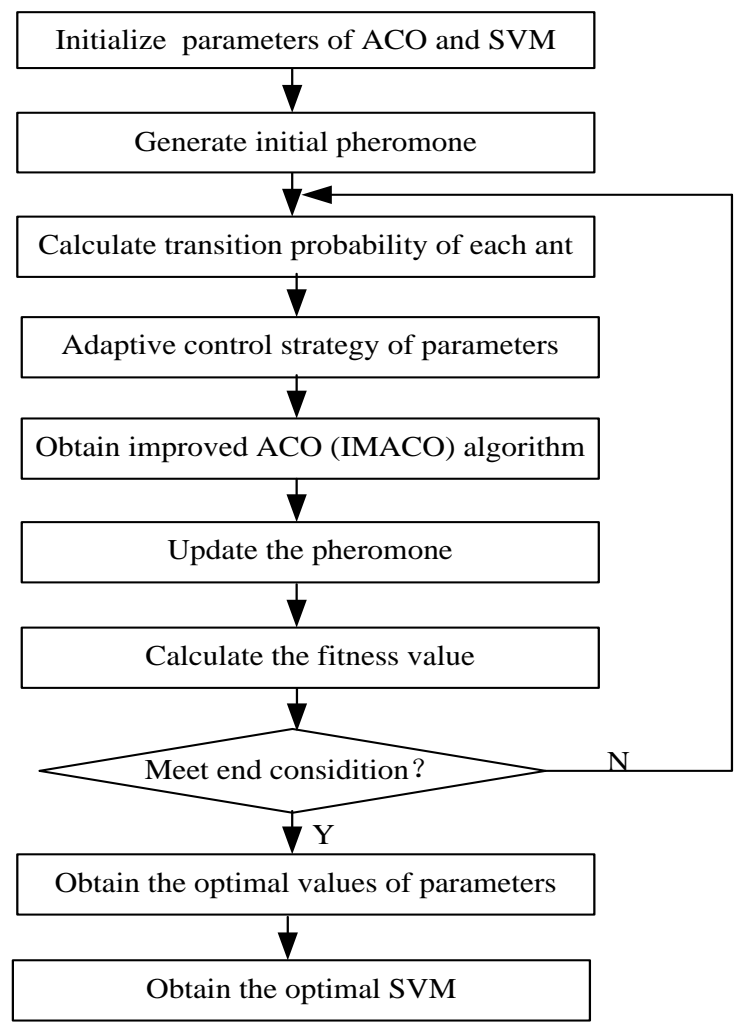

Figure 2. The Optimization Process of SVM Classifier

\subsection{A Fault Diagnosis Method Based on the Improved ACO and SVM}

The fault diagnosis has been receiving more and more attention and various fault diagnosis methods have been proposed in the past two decades. For small-scale system, these fault diagnosis methods takes on stronger diagnosis ability. But for the large-scale system, the existed fault diagnosis methods still cannot effectively achieve fault diagnosis. And the proposed optimal SVM model takes on better generalization ability, it is used to realize the fault diagnosis. So a new fault diagnosis (IMASFD) method based on the improved ACO and SVM is proposed in this paper. The framework of IMASFD method is shown in Figure 3. 


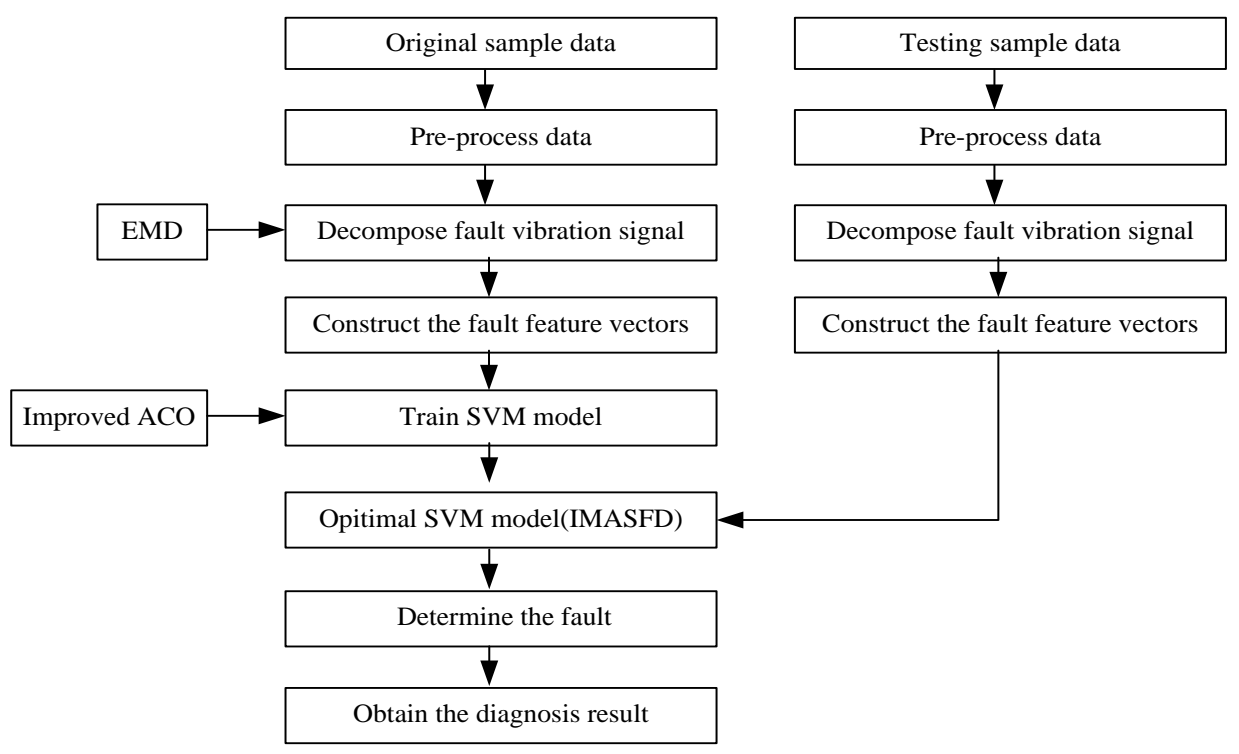

Figure 3. The Framework of IMASFD Method

\section{Bearing Fault Diagnosis Analysis}

In order to verify the validity and practicability of the proposed fault diagnosis (IMASFD) method, several common faults of rolling bearings are analyzed by the experiment. The vibration data came from Bearing Data Center of Case Western Reserve University. The 6205-2RS JEM SKF deep groove ball bearing is employed in the experiment. In this bearing, the internal diameter is $25 \mathrm{~mm}$, the external diameter is $52 \mathrm{~mm}$ and thickness is $15 \mathrm{~mm}$. The corresponding three faults(inner-race fault, outerrace fault and rolling element fault) are simulated by grooving on the inner ring and the outer ring and rolling of bearing using electric spark machining mode. As shown in Fig. 3 , the test bed consists of $1.5 \mathrm{~kW}$ motor, torque sensor/encoder, dynamometer, and electrical control devices. The motor drives the input shaft and the output shaft drives the load. The vibration data are sampled under different working conditions: for motor loads of 0 to 3 horsepower(HP). In addition, the data of different fault states are acquired with the sampling frequency of $12 \mathrm{kHz}$. The sampled original vibration signal is divided into a data sample with 4096 data points. The vibration signals on the innerrace and the outer-race and rolling of bearing under three states of faults respectively are 40 samples, and the vibration signals under normal state are 40 samples.

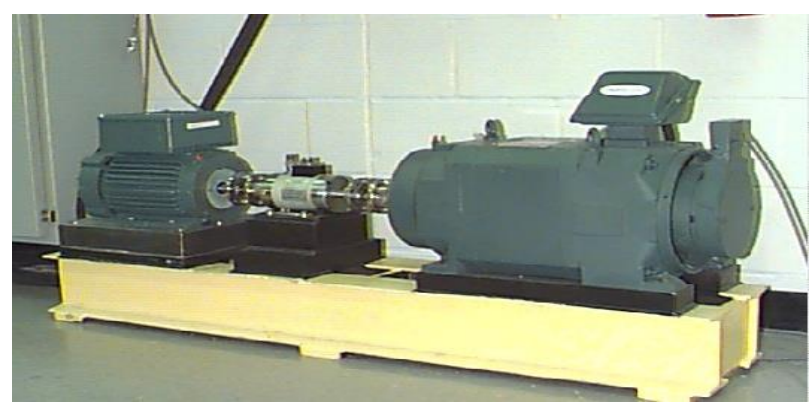

Figure 4. Test Bed

In order to effectively diagnose bearing fault patterns, three kinds of fault vibration signals respectively are decomposed by using empirical mode decomposition (EMD). The amplitude energy of ahead eight IMF components of three kinds of fault signals is extracted as the feature vector of representation fault patterns. The ahead eight IMF 
components of bearing under each working state are calculated and the instantaneous amplitude energy is calculated. And the instantaneous amplitude energy is regarded as input feature vector of IMASFD method, shown in Tab.1. A portion of the samples only are given due to space limitations. The obtained the feature vectors of bearing with four states are randomly divided into training samples (20) and testing samples(20). The training samples are used to construct fault diagnosis model based on improved ACO algorithm. The testing samples is used to test recognition accuracy of constructed fault diagnosis model.

Table 1. Fault Feature Vectors of Bearing

\begin{tabular}{cccccccccc}
\hline \multirow{2}{*}{ Working state } & Samples & \multicolumn{7}{c}{ The instantaneous amplitude energy features of IMF components } \\
\cline { 2 - 10 } & & $E_{1}$ & $E_{2}$ & $E_{3}$ & $E_{4}$ & $E_{5}$ & $E_{6}$ & $E_{7}$ & $E_{8}$ \\
\hline \multirow{2}{*}{ Rolling } & 1 & 0.722 & 0.630 & 0.242 & 0.150 & 0.033 & 0.005 & 0.002 & 0.002 \\
element fault & 2 & 0.751 & 0.593 & 0.264 & 0.116 & 0.029 & 0.003 & 0.002 & 0.001 \\
& 3 & 0.774 & 0.559 & 0.287 & 0.091 & 0.025 & 0.002 & 0.001 & 0.000 \\
Inner-race fault & 1 & 0.961 & 0.252 & 0.103 & 0.050 & 0.019 & 0.006 & 0.001 & 0.000 \\
& 2 & 0.950 & 0.288 & 0.104 & 0.052 & 0.019 & 0.004 & 0.002 & 0.000 \\
Outer-race fault & 3 & 0.942 & 0.304 & 0.105 & 0.055 & 0.018 & 0.003 & 0.003 & 0.001 \\
& 1 & 0.742 & 0.662 & 0.106 & 0.027 & 0.009 & 0.001 & 0.001 & 0.000 \\
& 2 & 0.708 & 0.699 & 0.103 & 0.023 & 0.006 & 0.002 & 0.000 & 0.000 \\
Normal & 3 & 0.684 & 0.723 & 0.102 & 0.021 & 0.003 & 0.003 & 0.002 & 0.000 \\
& 1 & 0.513 & 0.706 & 0.106 & 0.253 & 0.396 & 0.077 & 0.005 & 0.003 \\
& 2 & 0.527 & 0.713 & 0.189 & 0.260 & 0.329 & 0.054 & 0.009 & 0.006 \\
& 3 & 0.535 & 0.721 & 0.238 & 0.274 & 0.296 & 0.040 & 0.112 & 0.007 \\
\hline
\end{tabular}

In order to prove the fault diagnosis correctness of proposed IMASFD method, the SVMFD and ACO-SVMFD methods are select to compare with IMASFD method. The environments are followed: the Pentium CPU $2.20 \mathrm{GHz}, 2.0 \mathrm{~GB}$ RAM with the Windows XP operating system, Matlab2012b. Because the initial values of parameters in these methods could seriously affect the diagnosis result, the most reasonable initial values of these parameters are obtained by testing and modifying. The obtained initial values of these parameters are: ants $m=30$, pheromone factor $\alpha=1.0$, evaporation factor $\rho=0.05$, heuristic factor $\beta=2.0$, pheromone amount $Q=50$, initial uniform probability $q_{\min }=0.05$, and $q_{\max }=1$, maximum iteration $T_{\max }=1000$. The comparison of fault diagnosis results is shown in Table 2.

Table 2. The Comparison of Fault Diagnosis Results

\begin{tabular}{cccc}
\hline \multirow{2}{*}{ Working state } & \multicolumn{3}{c}{ Diagnosis correctness rate(\%) } \\
\cline { 2 - 4 } & SVMFD & ACO-SVMFD & IMASFD \\
\hline Rolling element fault & $89.34 \%$ & $92.46 \%$ & $94.93 \%$ \\
Inner-race fault & $89.72 \%$ & $93.26 \%$ & $96.04 \%$ \\
Outer-race fault & $91.36 \%$ & $96.67 \%$ & $99.74 \%$ \\
Normal & $92.47 \%$ & $97.26 \%$ & $100.00 \%$ \\
\hline
\end{tabular}

As can be seen from Tab.2, for the given testing samples, the fault diagnosis rate of SVMFD method for rolling element fault, inner-race fault, outer-race fault and normal respective are $89.34 \%, 89.72 \%, 91.36 \%$ and $92.47 \%$. The fault diagnosis rate of ACO- 
SVMFD method for rolling element fault, inner-race fault, outer-race fault and normal respective are $92.46 \%, 93.26 \%, 96.67 \%$ and $97.26 \%$. The fault diagnosis rate of proposed IMASFD method for rolling element fault, inner-race fault, outer-race fault and normal respective are $94.93 \%, 96.04 \%, 99.74 \%$ and $100.00 \%$. The comparison results show that the improved ACO algorithm can better obtain the global optimal parameters of fault diagnosis model than basic ACO algorithm. So the proposed IMASFD method has higher fault pattern recognition accuracy of bearing.

\section{Conclusion}

In this paper, an improved ACO algorithm based on adaptive control parameters and SVM model are used to improve fault diagnosis correctness rate and decrease misinformation diagnosis. We proposed a new fault diagnosis(IMASFD) model and method. Firstly, the EMD method is used to decompose fault vibration signal into IMF components, the energy of IMF components is selected to construct the fault feature vectors with reaction fault features. Then the adaptive controlling pheromone, adaptive controlling stochastic selection threshold and dynamic evaporation rate are used to improve ACO algorithm, which is used to optimize the kernel parameter $\sigma$ and penalty coefficient $C$ of SVM model, in order to obtain the optimal values of parameter combination. And a high-precision SVM model based on improved ACO algorithm is proposed in order to propose a new fault diagnosis(IMASFD) method. The test data from Bearing Data Center of Case Western Reserve University show that the proposed IMASFD method can accurately and effectively realize high precision fault diagnosis of rolling bearing, and has strong robustness and generalization ability, provides an effective method for realizing fault diagnosis of rolling bearing.

\section{Acknowledgements}

This research was supported by the National Natural Science Foundation of China (51475065, U1433124), the Open Project Program of State Key Laboratory of Mechanical Transmissions (Chongqing University)(SKLMT-KFKT-201416), the Open Project Program of the Traction Power State Key Laboratory of Southwest Jiaotong University (TPL1403), Open Project Program of Guangxi Key laboratory of hybrid computation and IC design analysis(HCIC201402), Open Project Program of Provincial Key Laboratory for Computer Information Processing Technology, Soochow University (KJS1326), the Open Project Program of Sichuan Provincial Key Lab of Process Equipment and Control(GK201404), the Open Project Program of Artificial Intelligence Key Laboratory of Sichuan Province (Sichuan University of Science and Engineering) (2014RYJ01). The program for the initialization, study, training, and simulation of the proposed algorithm in this article was written with the tool-box of MATLAB 2010b produced by the Math-Works, In

\section{References}

[1] J.D. Wu and C.H. Liu, "An expert system for fault diagnosis in internal combustion engines using wavelet packet transform and neural network. Expert System and Application”, vol. 36, no. 3, (2009), pp. 4278-4286.

[2] P.W. Ilott and A.J. Griffiths, "Fault diagnosis of pumping machinery using artificial neural networks. Proceedings of the Institution of Mechanical Engineers", Part E: Journal of Process Mechanical Engineering, vol. 211, no. 3, (1997), pp. 185-194.

[3] Z.Q. Geng and Q.X. Zhu, "Rough set-based heuristic hybrid recognizer and its application in fault diagnosis", Expert System and Application, vol. 36, no. 2, pp. 2711-2718, (2009).

[4] A. Chrissanthi, "Online expert systems for fault diagnosis in technical processes", Expert Systems, vol. 25, no. 2, (2008), pp. 115-132.

[5] H.L. Liu, H.X. Pan and A.Y., "Rough sets algorithm and its application in fault diagnosis", Telkomnika, Indonesian Journal of Electrical Engineering, vol. 11, no. 9, (2013), pp. 5471-5479. 
[6] P. Nguyen, M. Kang, J.Y. Kim and J.M. Kim, "Reliable fault diagnosis of low-speed bearing defects using a genetic algorithm", Lecture Notes in Computer Science, vol. 8862, (2014), pp. 248-255.

[7] S.F. Yuan and F.L. Chu, "Support vector machines-based fault diagnosis for turbo-pump rotor", Mechanical Systems and Signal Processing, vol. 20, no. 4, (2006), pp. 9393-952.

[8] S.F. Yuan and F.L. Chu, "Fault diagnosis based on support vector machines with parameter optimisation by artificial immunisation algorithm", Mechanical Systems and Signal Processing, vol. 21, no. 3, (2007), pp. 1318-1330.

[9] A. Widodo and B.S. Yang, "Wavelet support vector machine for induction machine fault diagnosis based on transient current signal", Expert Systems with Applications, vol. 35, no. 12, (2008), pp. $307-$ 316.

[10] S.W. Fei and X.B. Zhang, "Fault diagnosis of power transformer based on support vector machine with genetic algorithm”, Expert Systems with Applications, vol. 36, no. 8, (2009), pp. 11352-11357.

[11] Y.X. Zhang and R.B. Randall, "Rolling element bearing fault diagnosis based on the combination of genetic algorithms and fast kurtogram", Mechanical Systems and Signal Processing, vol. 23, no. 5, (2009), pp. 1509-1517.

[12] X.N. Lin, S.H. Ke, Z.T. Li, H.L. Weng and X.H. Han, "A fault diagnosis method of power systems based on improved objective function and genetic algorithm-tabu search, IEEE Transactions on Power Delivery, vol. 25, no. 3, (2010), pp. 1268-1274.

[13] A. Azadeh, M. Saberi, A. Kazem, V. Ebrahimipour, A. Nourmohammadzadeh and Z. Saberi, "A flexible algorithm for fault diagnosis in a centrifugal pump with corrupted data and noise based on ANN and support vector machine with hyper-parameters optimization", Applied Soft Computing Journal, vol. 13, no. 3, (2013), pp. 1478-1485.

[14] F.F. Chen, B.P. Tang and R.M. Chen, "A novel fault diagnosis model for gearbox based on wavelet support vector machine with immune genetic algorithm", Measurement: Journal of the International Measurement Confederation, vol. 46, no. 1, (2013), pp. 220-232.

[15] X.Y. Zhang and J.Z. Zhou, "Multi-fault diagnosis for rolling element bearings based on ensemble empirical mode decomposition and optimized support vector machines", Mechanical Systems and Signal Processing, vol. 41, no. 1-2, (2013), pp. 127-140.

[16] Y.S. Wang, Q.H. Ma, Q. Zhu, X.T. Lium and L.H. Zhao, "An intelligent approach for engine fault diagnosis based on Hilbert-Huang transform and support vector machine", Applied Acoustics, vol. 75, no. 1, (2014), pp. 1-9.

[17] D.C. Yao, L.M. Jia, Y. Qin and J.W. Yang, "Faults diagnosis of railway rolling bearing by using time-frequency feature parameters and genetic algorithm neural network", Computer Modelling and New Technologies, vol. 18, no. 10, (2014), pp. 441-445.

[18] F.F. Chen, B.P. Tang, T. Song and L. Li, "Multi-fault diagnosis study on roller bearing based on multi-kernel support vector machine with chaotic particle swarm optimization", Measurement: Journal of the International Measurement Confederation, vol. 47, no. 1, (2014), pp. 576-590.

[19] L. Pellaco, P. Costamagna, A. De Giorgi, A. Greco, L. Magistri,G. Moser and A. Trucco, "Fault diagnosis in fuel cell systems using quantitative models and support vector machines", Electronics Letters, vol. 50, no. 11, (2014), pp. 824-826.

[20] Z.Q. Su, B.P. Tang, Z.R. Liu and Y. Qin, "Multi-fault diagnosis for rotating machinery based on orthogonal supervised linear local tangent space alignment and least square support vector machine.Neurocomputing, vol. 157, (2015), pp. 208-222.

[21] R. Jegadeeshwaran and V. Sugumaran, "Fault diagnosis of automobile hydraulic brake system using statistical features and support vector machines", Mechanical Systems and Signal Processing, vol. 5253, no, 1, (2015), pp. 436-446.

[22] L. Lu, J.H. Yan, S. De Silva and W. Clarence, "Dominant feature selection for the fault diagnosis of rotary machines using modified genetic algorithm and empirical mode decomposition", Journal of Sound and Vibration, vol. 344, (2015), pp. 464-483.

[23] M. Kang, J. Kim, J.M. Kim, A. Tan, C.C. Andy, E.Y. Kim and B.K. Choi, "Reliable fault diagnosis for low-speed bearings using individually trained support vector machines with kernel discriminative feature analysis", IEEE Transactions on Power Electronics, vol. 30, no. 5, pp. 2786-2797.

[24] D.L. Yang, Y.L. Liu, S.B. Li, X.J. Li, L.Y. Ma. Gear fault diagnosis based on support vector machine optimized by artificial bee colony algorithm. Mechanism and Machine Theory, vol. 90, (2015), pp. 219-229.

[25] M. Dorigo and M. Gambardellal, "Ant colony system: A cooperative learning approach to the traveling salesman problem”, IEEE Transactions on Evolutionary Computation,vol. 1, no. 1, (1997), p. 53-66.

[26] V. Vapnik, "Statistical Learning Theory", New York: John Wiley \& Sons, (1998), pp.253-256.

[27] Y.F. Sun, P.C. Zhang and J. Qi, "A cognitive networks state prediction algorithm based on SVM improved by weighted RBF kernel function", Advances in Information Sciences and Service Sciences, vol. 4, no. 2, (2012), pp. 81-88.

[28] M. Vanny, K.E. Ko, S.M. Park and K.B. Sim, "Physiological responses-based emotion recognition using multi-class SVM with RBF Kernel.Journal of Institute of Control", Robotics and Systems, vol. 19, no. 4, (2013), pp. 364-371. 
[29] B.C. Kuo, H.H. Ho, C.H. Li, C.C. Hung and J.S. Taur, "A kernel-based feature selection method for SVM with RBF kernel for hyperspectral image classification", IEEE Journal of Selected Topics in Applied Earth Observations and Remote Sensing, vol. 7, no.1, (2014), pp. 317-326.

\section{Authors}

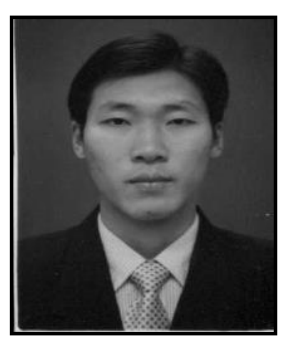

Wu Deng, Associate Professor, He received the Doctor degree in computer science and technology from Dalian Maritime University in 2012. His research interests: Artificial intelligence, Fault diagnosis, Computer application.

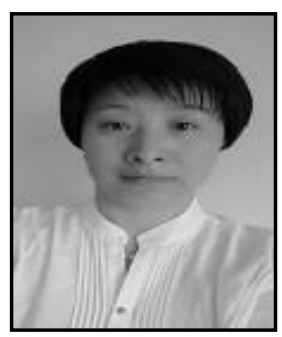

Xiumei Li, Lecture, She received the Master degree in computer science and technology from Dalian University of Technology in 2005. Her research interests: Artificial intelligence, Fault diagnosis.

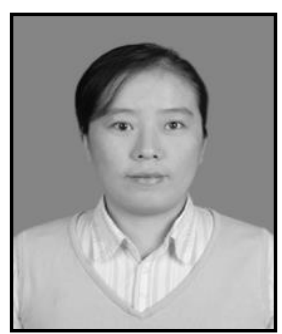

Huimin Zhao, Associate Professor, She received the Doctor degree in mechanical engineering and automation from Dalian Jiaotong University in 2013. Her research interests: Artificial intelligence, Signal processing, Fault diagnosis. 